\title{
Sentinel lymph node biopsy in medullary thyroid microcarcinoma using methylene blue dye mapping - a pilot study -
}

Authors:

Radan Dzodic 1,2, Nada Santracl, Merima Goran', Marko Butal',2, Igor Djurisicl, Gordana Pupic 3 , Ognjen Zivkovic ${ }^{3}$, Ivan Markovic ${ }^{1,2}$

1 Institute for Oncology and Radiology of Serbia, Surgical Oncology Clinic, Pasterova 14, Belgrade, Serbia 2 University of Belgrade, School of Medicine, Dr Subotica 8, Belgrade, Serbia

${ }^{3}$ Institute for Oncology and Radiology of Serbia, Department of Pathology, Pasterova 14, Belgrade, Serbia

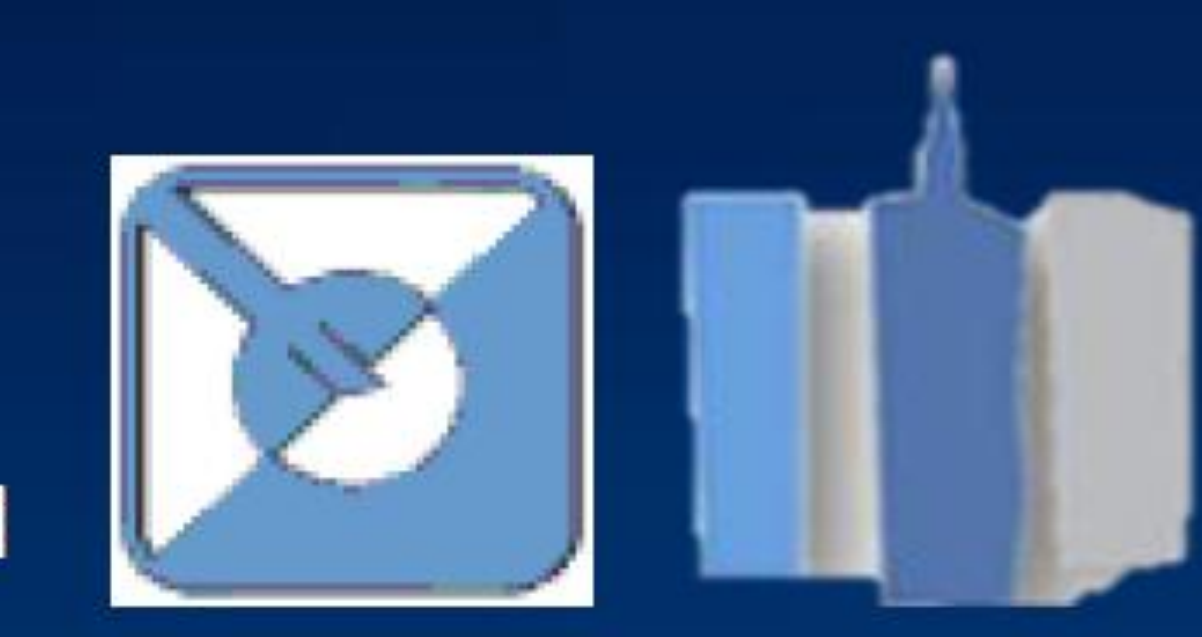

\section{INTRODUCTION}

Serum calcitonin level (sCT) is a precise marker for medullary thyroid carcinoma (MTC) [1]. However, lymph node (LN) metastases can be found in lower sCT, as well, and preoperative ultrasound may provide false negative findings on regional LNs $[2,3]$.

The aim was to present original technique of sentinel lymph node (SLN) biopsy of jugulo-carotid regions, after methylene blue dye mapping procedure, and its usefulness for selection of clinically NO patients with MTC for modified radical neck dissection (MRND).

\section{MATERIALS AND METHODS}

From 2007 to $2015^{\text {th }}, 14$ patients were operated in our Surgical Oncology Clinic due to MTC with sCT lower than $1000 \mathrm{pg} / \mathrm{ml}$, tumors under or $10 \mathrm{~mm}$ in size and clinically negative regional LNs. Central neck dissection was done in all patients. SLN mapping was performed with $0.2-0.5 \mathrm{ml}$ of $1 \%$-methylene blue dye [4]. Levels II and III were explored on both sides, blue stained SLNs (Figure) were removed and examined by frozen section analysis. If ex tempore result was negative (benign SLNS), additional surrounding non-colored LNs were removed for more precise evaluation and no further dissection was needed. If SLNs were positive (malignant), MRND was performed.

Figure: Blue-stained sentinel lymph

node identified in the right jugulocarotid region

\section{RESULTS}

One patient had hereditary form of MTC, with bilateral subcentimeter tumors, while others had sporadic, unilateral MTC. Sporadic MTCs showed no central nor lateral LN metastases on bilateral SLN biopsy, with no indication for MRND. Hereditary MTC had central LN metastases, with positive SLNs on both sides, thus one-time bilateral MRND was performed. This patient had metastases in other dissected LNs, as well, and SCT of 200 $\mathrm{pg} / \mathrm{ml}$. Frozen section and definite pathological analysis were $100 \%$ match.

\section{CONCLUSION}

SLN biopsy after methylene blue dye injection can be precisely used for intraoperative assessment of lateral LNs. It optimizes surgery for patients with MTCs, selecting clinically NO, but true positive patients for MRND. This pilot study is the first reported experience with SLN biopsy of jugulocarotid regions in MTC, using methylene blue dye.

\section{REFERENCES}

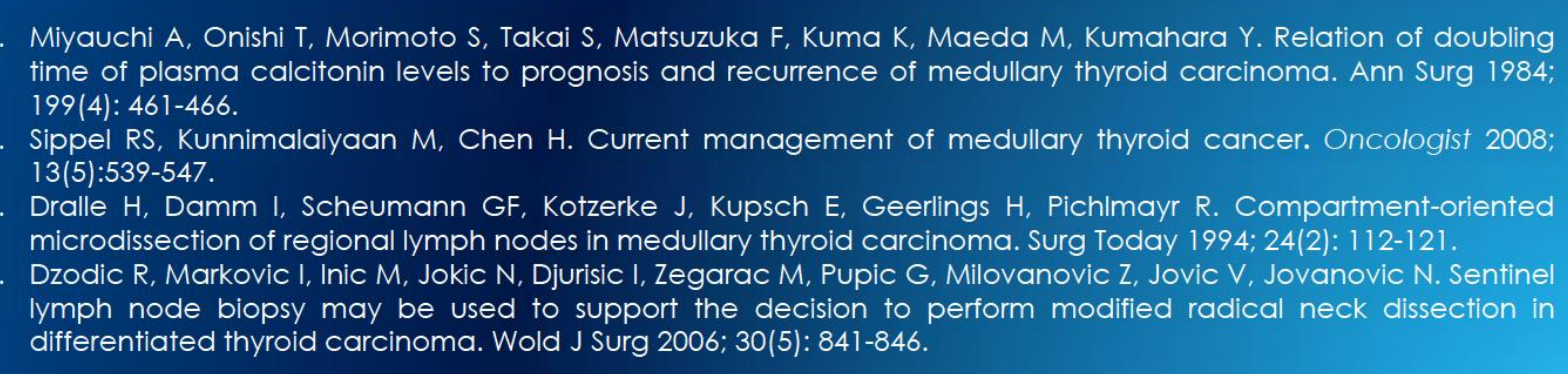

Endocrine tumors and neoplasia

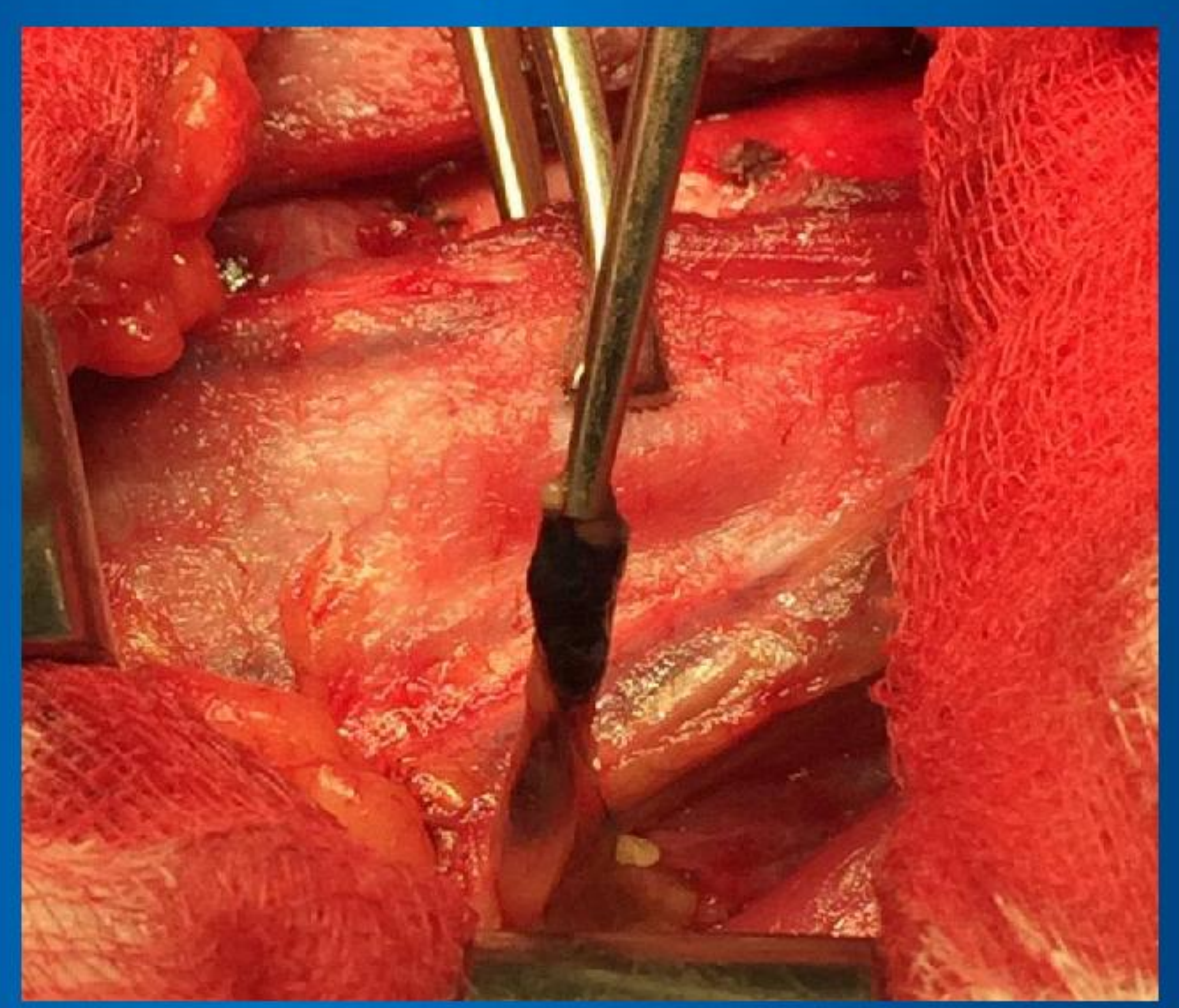

